\title{
Identifying and Mitigating Residual Vibrations in Wave-Based Control of Lumped, Flexible Systems
}

\author{
Hossein Habibi ${ }^{\text {a*}}$, William O'Connor ${ }^{\text {b }}$, S. Abolfazl Zahedi ${ }^{\mathrm{c}}$ \\ ${ }^{a}$ School of Science, Engineering \& Design, Teesside University, Tees Valley, Middlesbrough TS1 \\ 3BX, United Kingdom. \\ ${ }^{\mathrm{b}}$ UCD School of Mechanical and Materials Engineering, Engineering Building, UCD Belfield, Dublin \\ 4, Ireland. \\ ${ }^{c}$ School of Engineering and Sustainable Development, De Montfort University, Gateway House, \\ Leicester LE1 9BH, United Kingdom.
}

\begin{abstract}
Wave-based control (WBC) is a technique for motion control of under-actuated flexible systems. It envisages actuator motion as launching a motion wave into the system, while simultaneously absorbing any wave returning from the system. For rest-to-rest motion the net launch displacement is set at half the target displacement. In absorbing the returning wave and vibrations, WBC moves the system the remaining distance to the target, with zero steady-state error. The focus of this paper is on very small residual vibrations around the target position which can endure for a long time after arrival at target. This issue was discovered through a recent development within WBC context on controlling complex two-dimensional, mass-spring, beam-like arrays. To date their existence has been unidentified. This paper investigates and interprets the nature of these vibrations, explains and identifies them based on wave ideas, and finally offers a new wave-based approach to mitigate or suppress them. It also discusses their implication, not just for WBC but for the general problem of control of flexible systems.
\end{abstract}

\section{Keywords:}

Wave-based control, flexible system control, residual vibrations, vibration reduction.

\footnotetext{
*Corresponding author: e-mail: hossein.habibi@ucdconnect.ie
} 


\section{Introduction}

Wave-based control is a technique for motion control of under-actuated flexible systems. It envisages actuator motion as launching a motion wave into the system, while simultaneously absorbing any returning wave from the system. The term "wave" here describes a propagating disturbance, usually dispersing as it goes, which can leave behind a net change after it has entered and left the system via the actuator. For present purposes the wave variable can be taken as displacement, and, for rest-to-rest manoeuvres, the net change corresponds to the gross motion of the system. The interface between a grounded actuator and the flexible system is seen as a gateway for the interchange of motion between the actuator and the flexible system. It is a bi-directional gateway, with motion both entering and leaving the system simultaneously at every instant. To separate these two motions, the interface dynamics are monitored and resolved into counter-propagating motion components. The returning motion component is used for control. Figure 1 illustrates the idea. The actuator position, $x_{0}$, and the force, $f_{0}$, at the actuator/system interface (or equivalently, in the first spring) are measured. These are used to calculate the returning motion, in a way described elsewhere and can easily be implemented in real time (e.g. [1-3]).

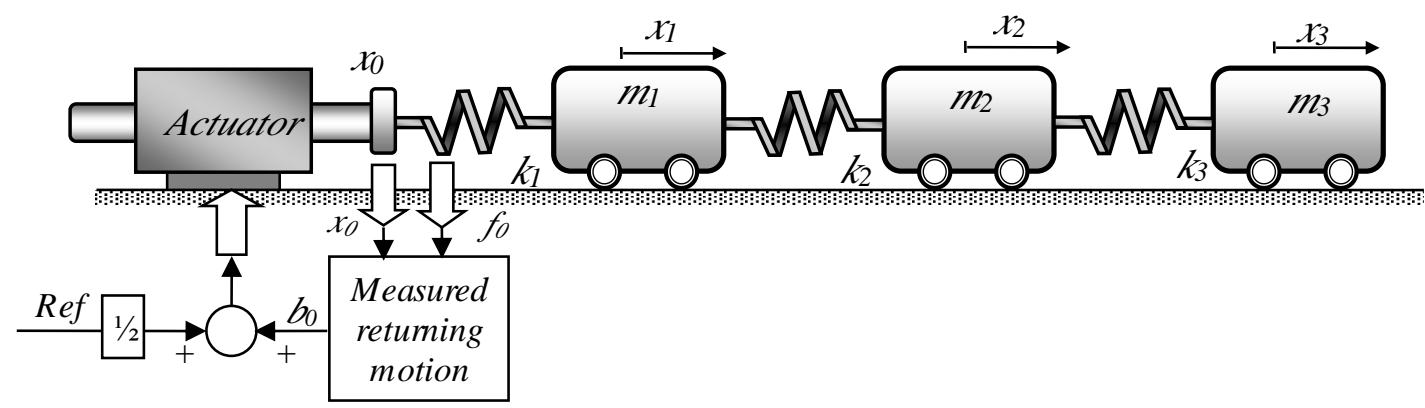

Fig.1. Wave-based, single-actuator control of 3-DOF mass-spring system.

The actuator is assumed to have its own position (sub-)controller, controlling $x_{0}$. As seen in the figure, the input to this actuator sub-controller is the sum of half the reference (or system target) position and the measured returning motion. The half-reference component can be considered as a motion wave which is launched into the flexible system by the actuator and which then travels to the system tip and back again. To this actuator motion is added the motion corresponding to the measured returning wave, which has the effect of absorbing it on its arrival back at the actuator. In fact, both launching and absorbing motions happen simultaneously, with the relative amounts of the two components changing continuously over time. 
The wave enters the system and leaves it again via the actuator, in general leaving behind a net displacement. In the absence of external disturbing forces, it can be proven that the net displacement associated with absorbing the returning wave, suitably defined and determined, will equal the net displacement associated with the launch wave which caused it. Absorbing the returning wave also provides active vibration damping. Thus the final rest position of the system will equal the final reference position exactly. Position control and active vibration damping are thereby seamlessly combined in a single actuator motion. Figure 2 shows an example of the response of a uniform three-mass system where the reference input is a steep ramp (steep in the context) to a target displacement of $1.0 \mathrm{~m}$.

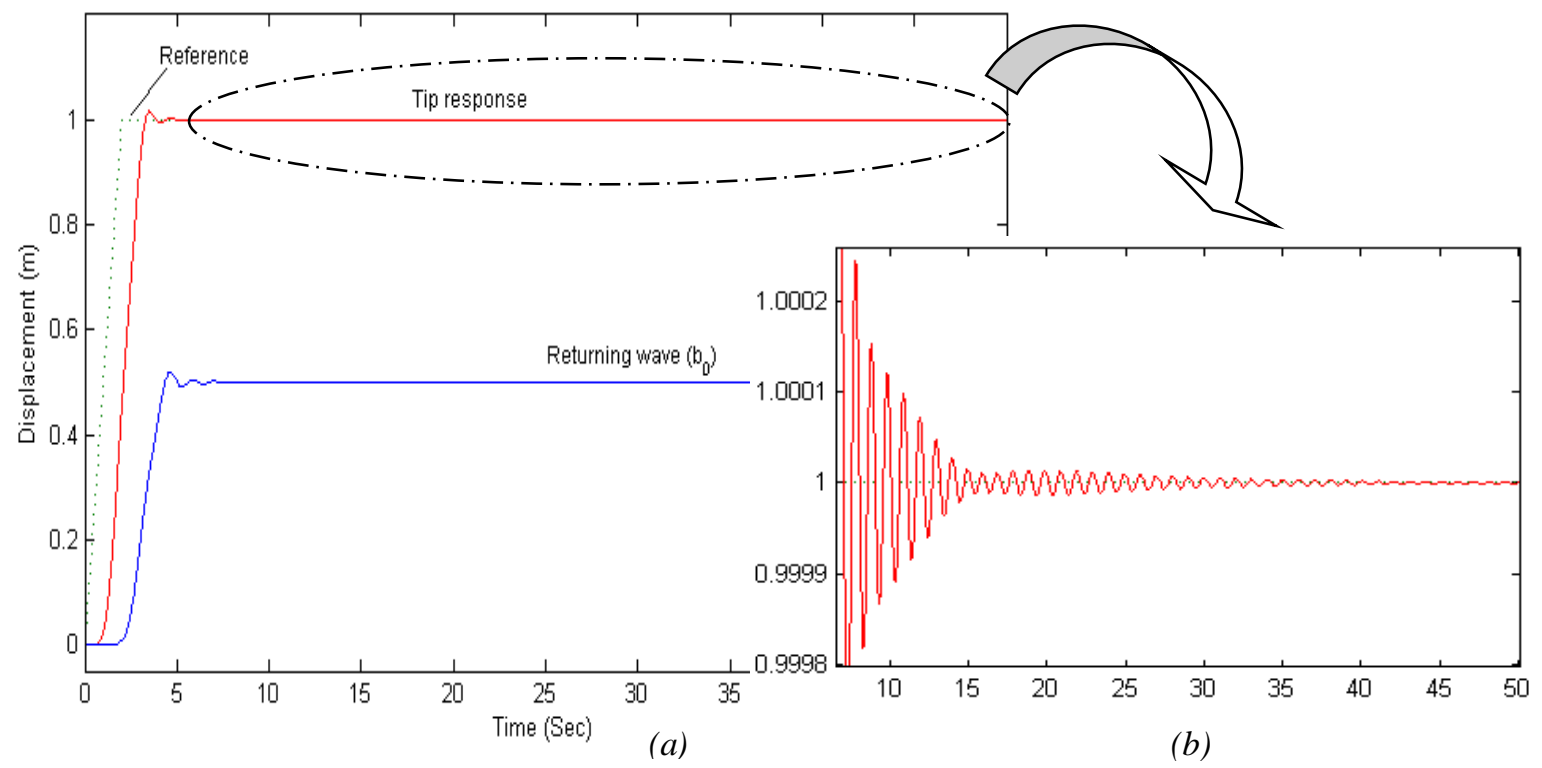

Fig.2. a) The tip response of the rectilinear system of Fig. 1. b) Magnification of the response shortly after arrival at target.

The system reaches the target rapidly and settles there, with no steady-state error. The blownup diagram however shows that there are very small residual oscillations centred on the target position which can endure a long time, in this case up to 10 times the main transient time, or about 50 seconds. Their amplitude in this case quickly falls below $10^{-3}$ times the net displacement and soon reaches $10^{-4}$. Textbooks typically define the settling time as the time after which the output remains within, say, $5 \%$ or $2 \%$ of the final value. Clearly the amplitude of these vibrations is of an order of magnitude smaller. But they are present. In fact this issue was revealed and drawn into attention through a recently published work [4] in which WBC was promoted to control translational and rotational motions of beam-like mass-spring structures in plane. As one moves towards more complex lumped flexible systems, such as these $2 \mathrm{D}$ flexible arrays of masses and springs, the residual vibrations under WBC become more evident, while 
generally remaining small. This prompted a closer investigation, to understand the nature of the effect, how it comes about, its significance, and how it might be reduced or eliminated.

But first a slightly wider context is appropriate. This is presented in Section 2. Section 3 then reviews aspects of wave-based control and wave models of lumped, flexible systems. Section 4 provides an explanation of the residual vibrations in terms of the wave models. Section 5 presents some sample results, for rectilinear flexible systems and 2-D mass-spring arrays under WBC. Section 6 considers that, rather than being associated with WBC alone, residual vibrations must be a wider issue. Finally Section 7 has some comments and conclusions.

\section{Context}

The control of under-actuated flexible systems has been studied for decades. There is an enormous literature on the topic, with a wide range of methods and applications. References [59] give some books and survey papers. It would neither be feasible nor appropriate to attempt a general summary here. Likewise it would take too long to put WBC into the context of these other approaches and to make detailed comparisons. Nevertheless, and at the risk of over-simplification, a very brief summary follows.

A number of reported flexible control strategies assume that a good model of the system is available, either because they are essentially open-loop (e.g. posicast, input shaping, time delay filtering, sliding mode and time-optimal bang-bang control) or the control is otherwise modelbased or model derived. For this reason these methods are generally not robust to modelling errors, unmodelled system behaviour, or unknown system changes. They may also have other challenges, such as chattering problems (in sliding mode) and implementation challenges (in time-optimal control). Other methods are based on modal analysis and modal control, working in the frequency domain. Difficulties can then arise associated with transient behaviour, mode measurement, mode truncation and mode spillover. Many methods, such as $\mathrm{H}_{\infty}$ and wave analysis, focus on the active vibration control aspects only, or considers them as separable from the motion control problem, as if happening in an inertial (non-accelerating) reference frame. Such separation of the motion into so-called rigid body and vibratory motion generally implies ignoring the inevitable cross-coupling between these notionally separated motions, or at best, regarding them as disturbances of one on the other. Furthermore such methods frequently assume small amplitude vibrations, even when allowing large gross motions. In addition, most of these methods ignore actuator limitations and assume that slow dynamic responses or saturation effects do not arise or are carefully avoided. 
By contrast, WBC circumvents all such problems. No system model is needed. There is no attempt to separate the motion into so called rigid-body and vibratory components, and so no concerns about coupling between them. It works in the time domain, avoiding any kind of modal analysis and its associated challenges and limitations. Furthermore the control system is stable, robust to system changes (known or unknown), robust to actuator dynamic limitations and actuator saturation, easily implemented, generic, and can achieve very rapid responses. Regarding the speed of response, if required WBC can achieve the appropriately-defined, timeoptimal response, by suitable fine-tuning of the launch waveform [10]. Further research on extensions to or applications of WBC has been published in, for example, [1-4]. Much of this work was tested using computer simulation and numerical models, but experimental work has also been carried out and reported on a variety of test rigs to verify the validity of the method practically in coping with simultaneous control of position and vibration [2, 11-12].

Regarding residual vibrations specifically, there is a sense in which all strategies for flexible system control are also about attempting to control residual vibrations. For example, see references [13-22]. In input shaping, for example, there is focus on making the controller more robust (or less sensitive) to departures of flexible system dynamics from nominal values. A metric of success then is the amplitude of the residual vibration as system frequencies vary from nominal, and in general the further from nominal the greater the amplitude. By contrast, the residual vibrations under WBC, considered here, are of a different nature, qualitatively and quantitatively. They are only remotely related to modelling errors, choice of input waveform, or implementation issues. They are observed as a) having high frequency, b) low amplitude for all input waveforms, c) are very slowly decaying, and d) are not decaying monotonically.

The theory of WBC guarantees that the residual vibrations must die out eventually, even without system damping, leaving the system at target. For many engineering purposes they are probably of little concern, especially because any internal damping will accelerate their decay. Nevertheless it is reasonable to ask how they arise; why they endure so long in the absence of damping; what determines their amplitude, frequency and decay rates; can they be actively reduced in the absence of passive damping; and are they unique to $\mathrm{WBC}$ ?

\section{Wave models for wave-based control}

Wave models of lumped flexible systems were developed to justify the wave analysis of lumped systems, and so wave-based control. The details of the wave models and their justification are considered in [23]. 


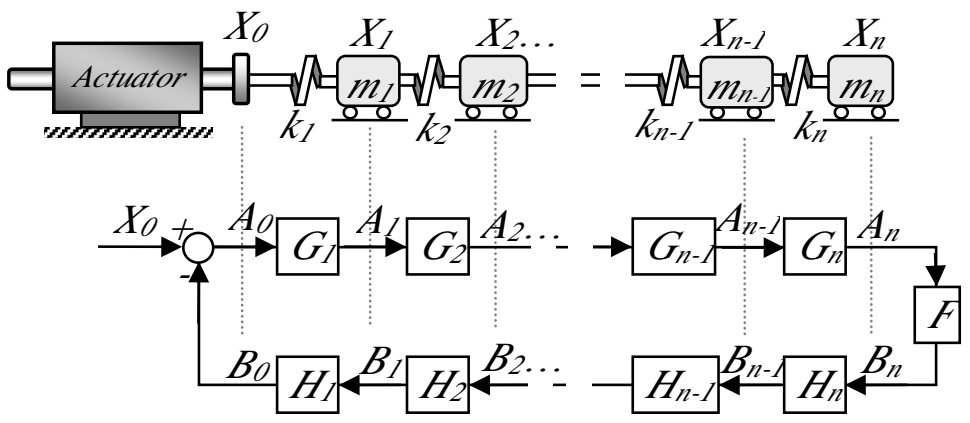

Fig.3. Series wave model (below) of the dynamics of a lumped mass-spring chain (above), with

$$
X_{i}=A_{i}+B_{i}
$$

Broadly, there is a series model, illustrated in Fig. 3 for the case of a rectilinear lumped massspring system, with an actuator at one end and the other end free. It involves a series of wave transfer functions (WTFs), $G_{\mathrm{i}}(\mathrm{s})$, in a loop as shown. The motion variables, $X_{i}$, in the physical system are seen as the superposition of the corresponding motions in the upper and lower branches of the wave model, or

$$
X_{i}(\mathrm{~s})=A_{i}(\mathrm{~s})+B_{i}(\mathrm{~s})
$$

The $A_{i}$ terms define the outgoing waves, the $B_{i}$ the returning waves. In particular, this model allows the actuator motion, $X_{0}$ to be resolved into outgoing and returning components, $A_{0}$ and $B_{0}$.

Likewise, $H_{i}$, in the lower branch, are transfer functions between successive masses in a string initially corresponding to the real system but followed to the left by an imagined extension to infinity. Thus

$$
\begin{aligned}
& A_{i}(s)=G(s) A_{i-1}(s) \\
& B_{i}(s)=H(s) B_{i+1}(s)
\end{aligned}
$$

At the free end, the two loops are joined by another transfer function, $F$, chosen to ensure that the superposition of $A_{n}$ and $B_{n}$ model the motion of the free end mass $m_{n}$. At the actuator end the boundary is modelled by a negative feedback loop. It can be shown [1] that the superposition of the outgoing and returning waves obeys the equations of motion of the original system and its boundary conditions. In other words, in the sense given by Eqs. (1) and (2), this "wave model" models the real system dynamics exactly.

If the system is uniform, then all the $G_{\mathrm{i}}(\mathrm{s})$ and $H_{\mathrm{i}}(\mathrm{s})$, as well as $F(\mathrm{~s})$ can be made equal, and the form of this wave transfer function (WFT) becomes 
where $\omega=\sqrt{\frac{k_{1}}{m_{1}}}$.

$$
G(s)=1+\frac{1}{2}\left(\frac{s}{\omega}\right)^{2}-\left(\frac{s}{\omega}\right) \sqrt{1+\left(\frac{s}{2 \omega}\right)^{2}}
$$

A second form of the wave model has wave shunt paths, as shown in the lower part of Fig.4 for a section of a mass-spring system shown above it. Again the upper path represents the outwards-going, rightwards $\left(A_{i}\right)$ waves, the lower branch the waves $\left(B_{i}\right)$ returning towards the actuator, with $X_{i}(\mathrm{~s})=A_{i}(\mathrm{~s})+B_{i}(\mathrm{~s})$. The actuator and free ends of this model are similar to those in the series model of Fig.3.
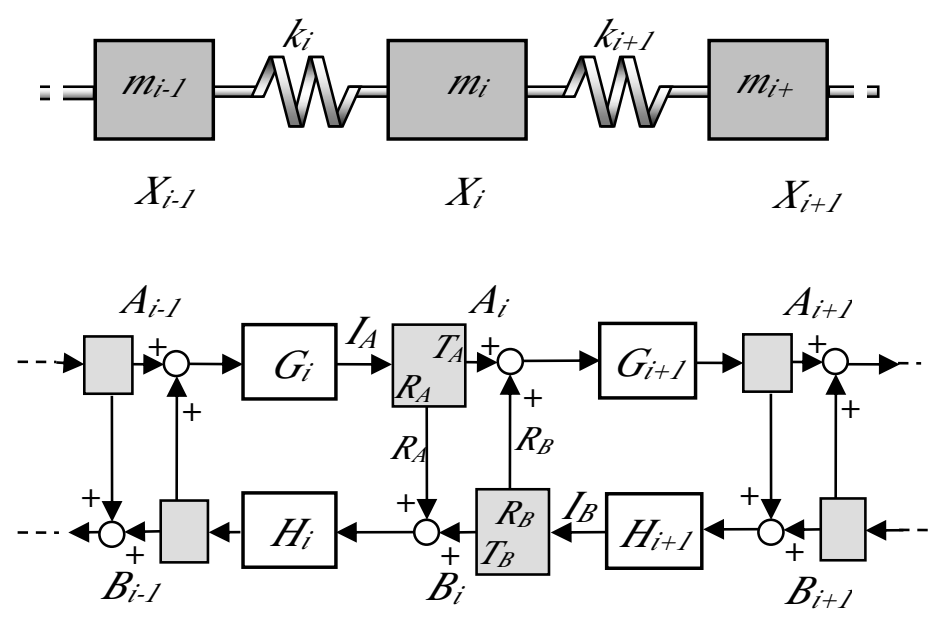

Fig.4 Shunt wave model (below) of mass-spring chain (above), with $X_{i}=A_{i}+B_{i}$

This form models partial transmission and partial reflection of waves at each mass, for both the outgoing and returning waves. The reflected part of an outgoing wave in the upper branch is shunted to the lower returning branch, and the opposite happens for returning waves. The calculation of the transmission and reflection coefficients is given in [23].

Both the series and shunt models model the lumped system dynamics exactly, in the sense that, when their components are properly defined, the sum of the outgoing and returning waves reproduces the dynamics of the lumped system exactly. The series and shunt configurations constitute alternative models of the same dynamical system. Also they are closely related. For example, it is possible to choose WTFs in the shunt model which cause the shunts to be unnecessary, thereby recovering the series model.

For WBC the main reason for the models is to allow the actuator's motion to be resolved into outgoing and returning component motions. For this, the two models lead to similar WBC implementations, as they model the interface between the actuator and the flexible system in 
similar ways. In particular, for both models, in the uniform case, the returning wave $B_{0}$ can be expressed as

$$
B_{0}(s)=G(s)\left(X_{1}(s)-G(s) A_{0}(s)\right)
$$

WBC attempts to absorb this returning wave. The actuator input becomes the sum of half the reference input plus the measured return wave, $B_{0}(\mathrm{~s})$, of Eq.(4), or $b_{0}(t)$, in the time domain, as shown in Fig.1. From the perspective of the wave models, this is equivalent to preventing the returning wave from being reflected at the actuator and so re-entering the system which, if allowed, would maintain the vibration. The wave model in Fig. 3 shows $-B_{0}$ being re-inserted into the system, which is what would happen in the absence of actuator control. Under WBC the actuator control adds a $B_{0}$ term to the actuator motion, $X_{0}$, which cancels the re-insertion and so absorbs the returning wave. It is equivalent to "opening the loop" at the left hand end of the series wave model in Fig.3, so that the waves launched by the actuator do a single round trip, permanently leaving the system on their return to the actuator, usually leaving behind a net displacement throughout the flexible system.

The practical implementation of the control system and the modelling of the $G(\mathrm{~s})$ transfer functions in Eq.(3) and (4) has been discussed in references [1-2, 24] and will not be considered here.

\section{A wave perspective on residual vibrations}

\subsection{Shunt wave model}

As mentioned, it can be proven that, under WBC, some time after the reference signal has reached the target position, the entire system will come to rest at the target with no steady-state error. One proof is based on the final value theorem [2]. The residual vibrations therefore must die out, sooner or later. But what happens in the meantime? Why the small residual vibrations before settling?

The wave models provide an explanation. It is clearest in the case of the shunt model. In a rest-to-rest manoeuvre, the main motion does indeed enter and leave the system, in such a way that, when it has left, the system has landed at target. However, in going through the system, every time the wave motion meets a new element, there is a partial transmission and partial reflection, for both the outgoing and returning waves. These reflected components undergo further scattering, which add to the primary scattering, in an on-going process, sometimes adding 
to already scattered components, sometimes tending to cancel. In this way smaller and smaller portions of the travelling waves become more and more scattered and dispersed within the lumped system.

The resulting vibrations have some of the features of standing waves of small amplitude and high frequency. But it is better to see them as travelling waves, continuing to propagate through the system, albeit following very circuitous routes, with smaller and smaller portions taking more and more circuitous routes. If, at each successive scattering point in a round trip, the transmitted components are larger than the reflected, the largest motion components will arrive back to the actuator most rapidly, with smaller and smaller components taking longer and longer. Eventually, after shorter or longer routes, components will find their way back to the actuator, where they will be absorbed by the action of the WBC arrangement. The WBC measures and absorbs the entire returning wave, as one superposed motion, regardless of the different path lengths of its components and the different elapsed times since they entered the system as part of an actuator launch wave.

This reasoning accounts for the main features of the residual vibration, namely small amplitude and very slow decay. The high frequency can be seen as a consequence of the frequency dependence of the transmission and reflection coefficients, or the increased wave impedance of the lumped system with higher frequencies. In the example in Fig.2, the frequency approaches $2 \omega_{n}$, that is, a little below the frequency at which there is a cut-off for propagation through a uniform lumped system, or the limit of the highest natural frequency of a fixed-free uniform vibrating system. Although the $\mathrm{WBC}$ is designed and implemented in the time domain, it can be viewed in the frequency domain as acting as a low pass filter, allowing the DC and lower frequency AC components to pass through quickly, but causing more and more delays to higher frequency components.

It might be objected that harmonic waves can propagate through such lumped systems without apparent dispersion or reflection, as is frequently assumed. But harmonic solutions implicitly assume steady-state conditions. By contrast, position control involves transient waves, especially when starting from rest or attempting to return to rest at the end of a manoeuvre. Thus for rapid position control, the transient is all important. Arguably transients are best seen in the time domain, and the time response is best interpreted in terms of propagation delays. 


\subsection{Series wave model}

It was stated above that the series model reproduces the system dynamics precisely and can form the basis for WBC. At first sight, however, the absence of shunt paths makes it more difficult to account for the residual vibrations. In fact, they can be accounted for from the form of the WTFs. For simplicity we will consider only the uniform case, where $G(\mathrm{~s})$ is given by Eq.(3).

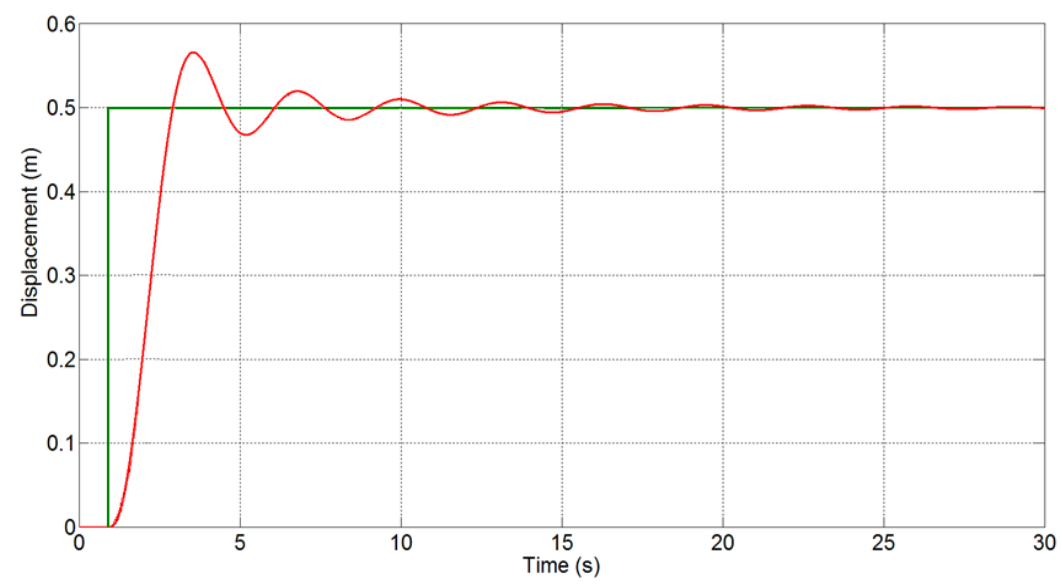

Fig.5 Time domain response of step input to the WTF of Eq. (3)

Figure 5 shows the exact response of this WTF to a step input. It is a Bessel function, which exhibits residual vibrations which last for a long time before eventually decaying to zero. In the series model, the overall response will involve a combination of such responses in series, that is, with the output of each one becoming the input to the next. Thus even the series wave model predicts, or accounts for, residual vibrations, which it must do, if it really is a valid model of the system.

The series model is not restricted to uniform systems, but in non-uniform systems the WTFs then become more complex than Eq.(3). Full details are given in [23]. Suffice to say that, in response to say a step input, the more complex WTFs display longer and more complex residual vibrations than for the uniform case above.

\subsection{Minimising residual vibrations}

The amplitude, nature, and decay rate of the residual vibrations depends both on the shape of the input waveform and on the dynamics of the flexible system.

It is certainly possible to move a lumped flexible system through a finite distance, from rest to rest, in such a way that on arrival at target there are no residual vibrations whatever. It can 
even be done rapidly, at least in theory, for example, using a time-optimal bang-bang, acceleration-limited input. If done perfectly, on arrival at target the system will be completely at rest. So the input waveform is certainly important.

Typically in WBC the input waveform is a step or ramp to target, as in Fig.1. But there is no reason not to use a specially designed input, such as one producing the time-optimal solution, if known. On the other hand, if a less specific input is used, the residual vibrations can be reduced by choosing a fairly generic input which is smoother and more symmetrical, for example by beginning with a suitable acceleration-coast-deceleration profile which is then integrated twice to get the displacement wave input.

Regarding the system itself, the more degrees of freedom the system has, the more scope there is for multiple wave paths, and so for more scattering and dispersion. This, in turn, will prolong the residual vibrations, as the components have more places to linger in the system and longer paths to return to the actuator. At the other extreme, with just one degree of freedom, there will be no recognizable residual vibrations, at least nothing like those shown in Fig.2.

Another factor is the degree of uniformity of the flexible system. A lumped system is inherently dispersive to waves, but the dispersion becomes more pronounced as the system becomes less uniform, for example with local changes in mass or spring values. In terms of wave scattering, the non-uniformity causes a reduction in the transmitted portion of the wave and an increase in the reflected portion. In other words, the scattering effects are increased.

\section{Sample results}

\subsection{Rectilinear systems}

To test the validity of this understanding and ideas, first a numerical model of a uniform three-mass 1-D (rectilinear) system similar to that in Fig.1 was tested. The masses were set to $1 \mathrm{~kg}$ and the spring stiffness to $10 \mathrm{~N} / \mathrm{m}$.

First, with a step input, or a low-pass filtered step input, there was a small but significant residual vibration which lasted for a long time after the system had arrived at the target displacement (similar to Fig.2). Significantly, filtering the input to make the waveform smoother (removing high frequency components) does not reduce the residual vibration. This confirms that the problem is not due simply to a lack of smoothness in the input. From the wave perspective, a step input can be considered as a very high acceleration followed by a very high deceleration, with no time for any wave-absorption or settling in between. 


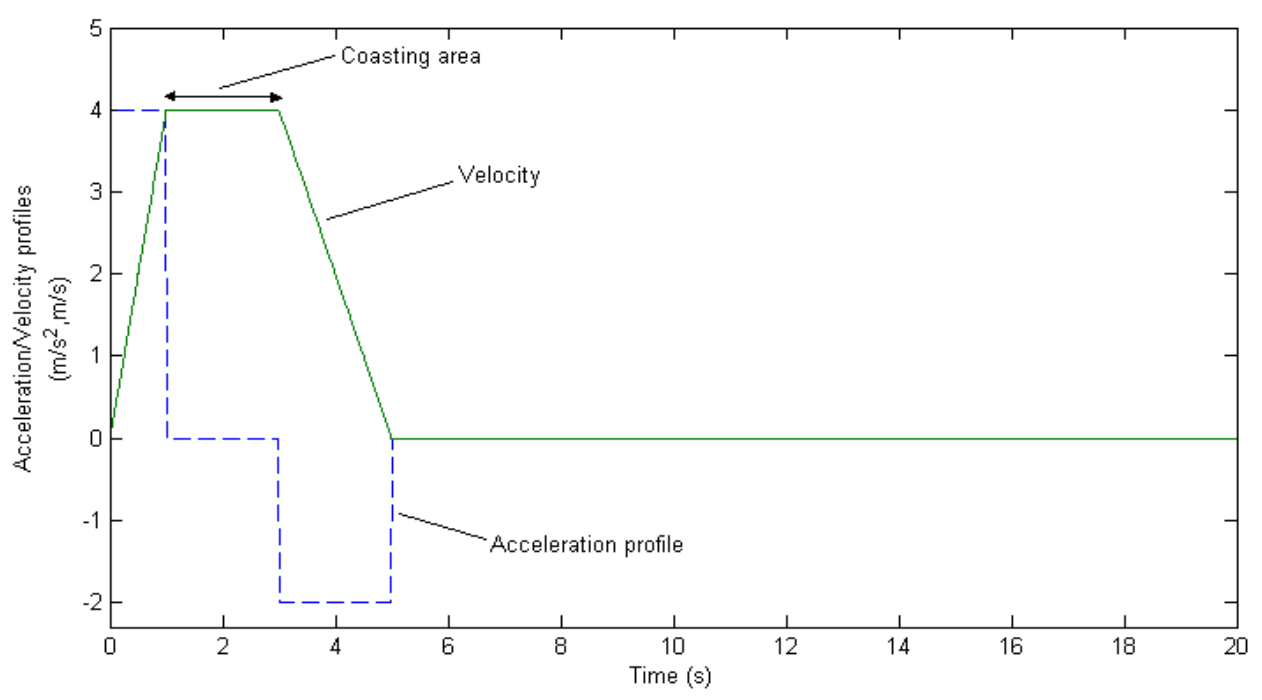

Fig.6. An input profile, comprising acceleration-coast-deceleration phases

Figure 6 shows an example of an input with an acceleration phase, a coasting phase, and a deceleration (negative acceleration) phase, as mentioned above. The response to this input is compared in Fig.7 to that obtained with a simple filtered ramp with a similar transit time and distance. The phased acceleration input produces a slightly better response. But the real benefit is seen in Fig.8 which compares the residual vibrations (on a highly magnified scale). A very significant reduction in the size and settling time of the residual vibration is evident.

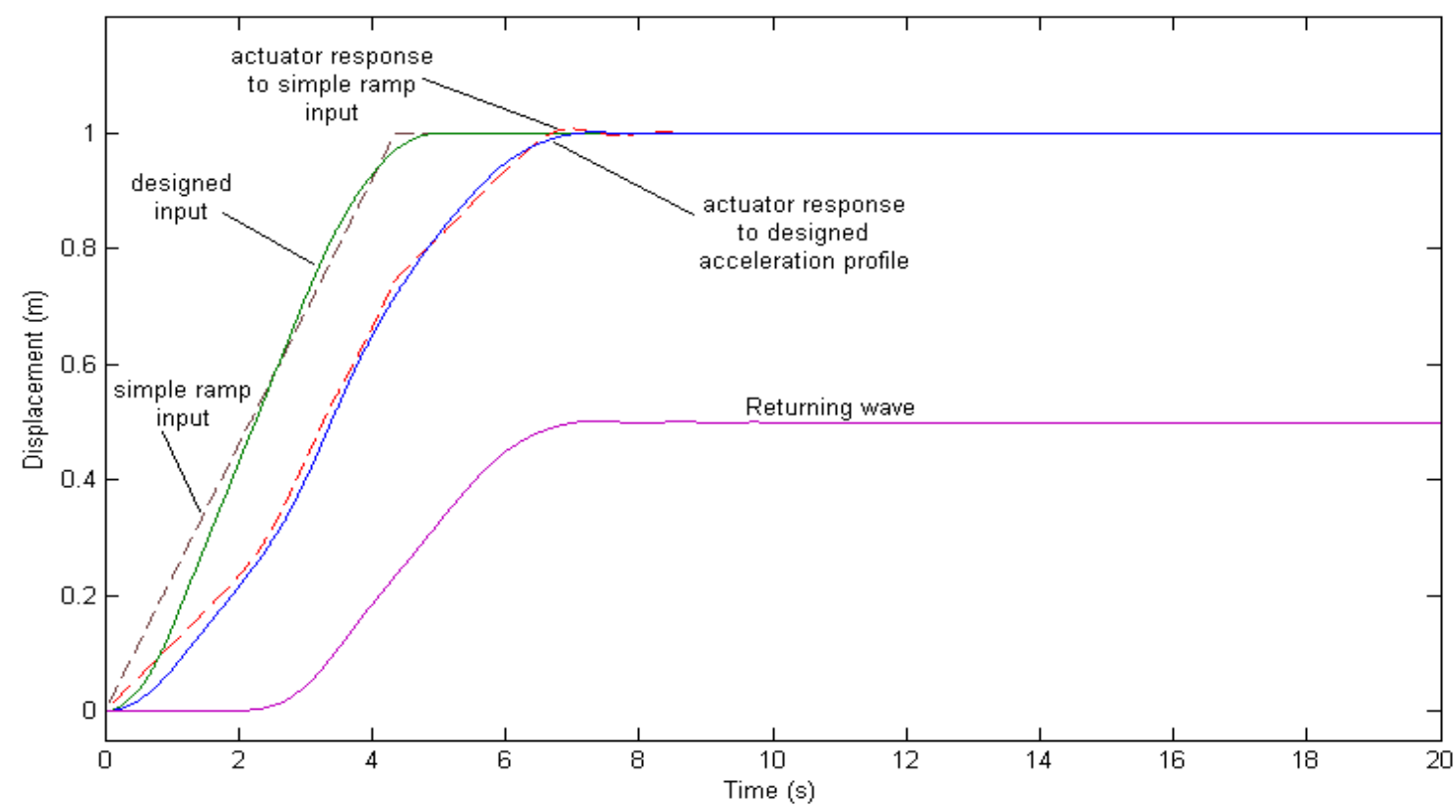

Fig.7. Comparing responses to inputs: designed acceleration profile versus simple ramp 


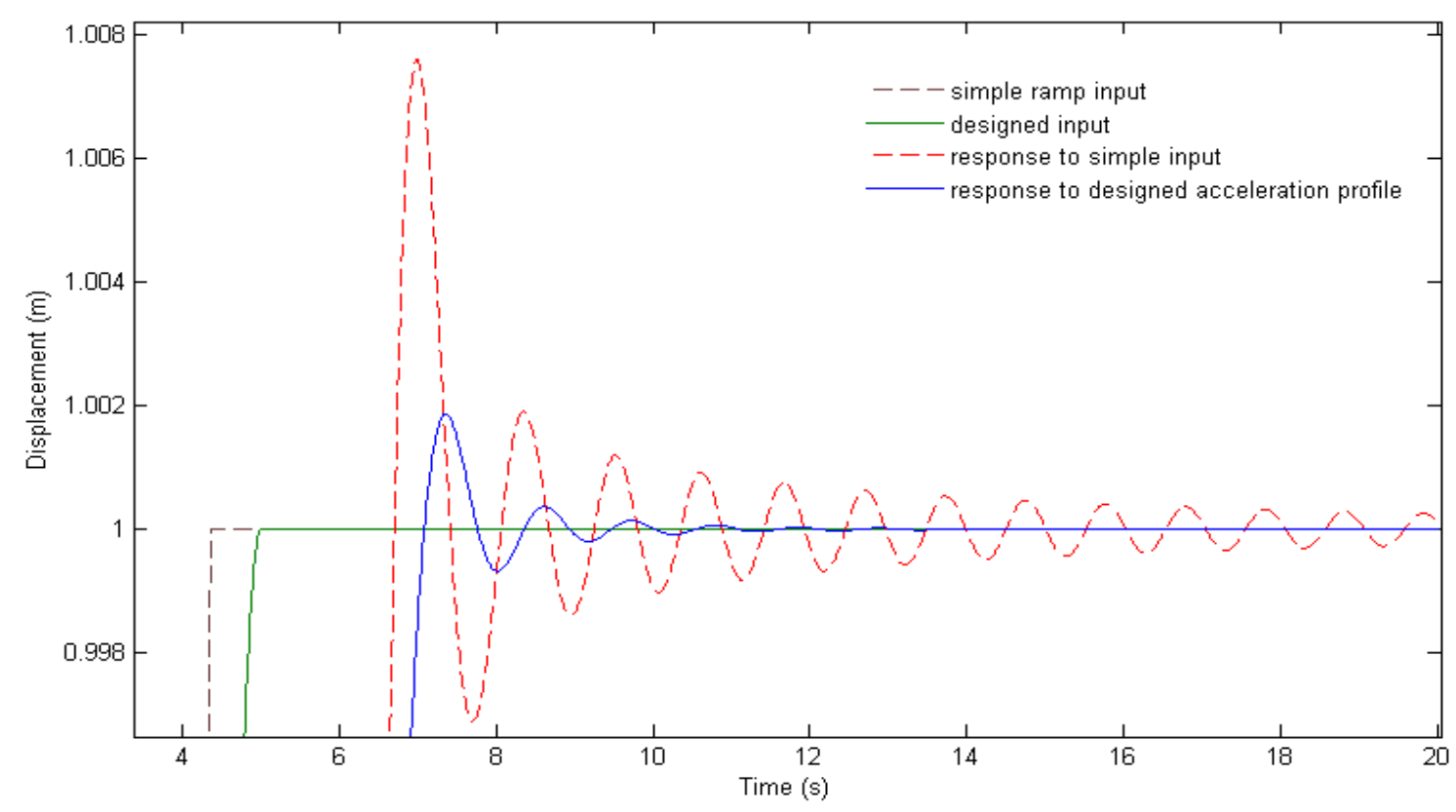

Fig.8. Residual vibrations with simple ramp and designed acceleration profile inputs

A significant point is that the neither the timing of the deceleration phase (with respect to the start time) nor its shape is critical. In Fig 6 for example, it can be seen that the acceleration and deceleration phases are not identical, but have the same net effect (same areas under curve, with opposite signs). The length of coasting phase, and so the timing of the deceleration phase, were also varied, and were found to give similar results. The coasting time should be long enough to smooth over residual oscillations in the measured interface force $\left(f_{0}\right)$. However, the required coasting time is not very long, and corresponds roughly to the time for a single wave to travel through the system, from actuator to tip and back again. In practice, a value slightly longer than one period of the lowest vibration mode of the flexible system works well for the coasting phase. The only important requirement is that the system should have a little time to settle (not perfectly of course) during the coasting phase. As for the acceleration and deceleration values, based on the requested displacement i.e. the actuator input, they are automatically calculated and set such that once they are integrated twice, the displacement wave input will be configured.

Clearly, this whole strategy implies a somewhat longer manoeuvre time (due to the time for the coasting phase) and a more gentle input.

These results now give the control designer a choice. If quickly reducing the small residual vibrations becomes a high priority, the strategy provides a way to do so. What is needed is not 
simply a low-pass filtered input, but something more strategic, which however is very simply implemented in practice.

\subsection{Two-dimensional systems: mass-spring arrays}

The ideas were then tested on the considerably more complex 2-D, planar, mass-spring array, the type of configuration which had initially drawn attention to the matter and prompted the investigation. The system has a single actuator as above, but now one which can both translate and rotate in the plane. Figure 9 is a schematic sketch of such a 2 -D system with its control system. The directly controlled actuator is at the bottom left hand corner, and it is indirectly controlling the attached flexible array, in which frequently the tip position and vibration would be of most interest.

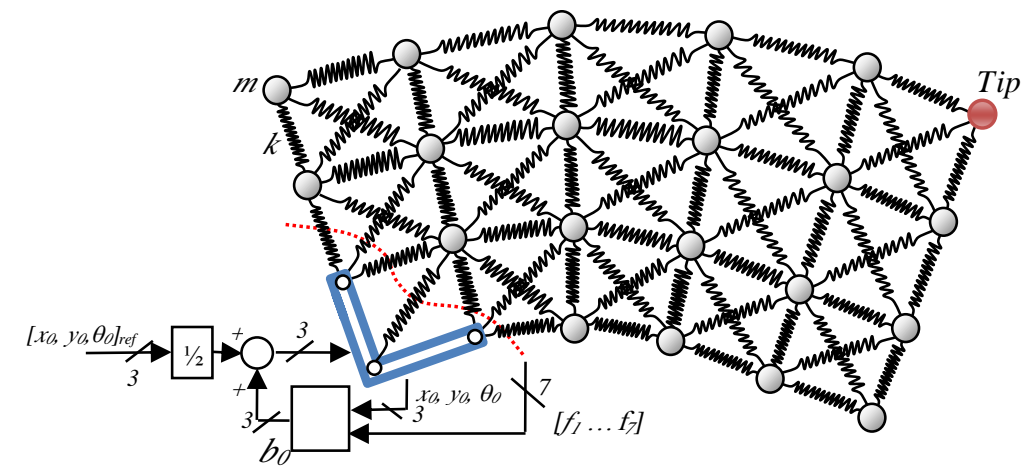

Fig.9. A typical 2-D lumped system under WBC

Reference values of translation and rotation $(x, y, \theta)$ can be inserted to displace the entire system from rest-to-rest at a target position and orientation.

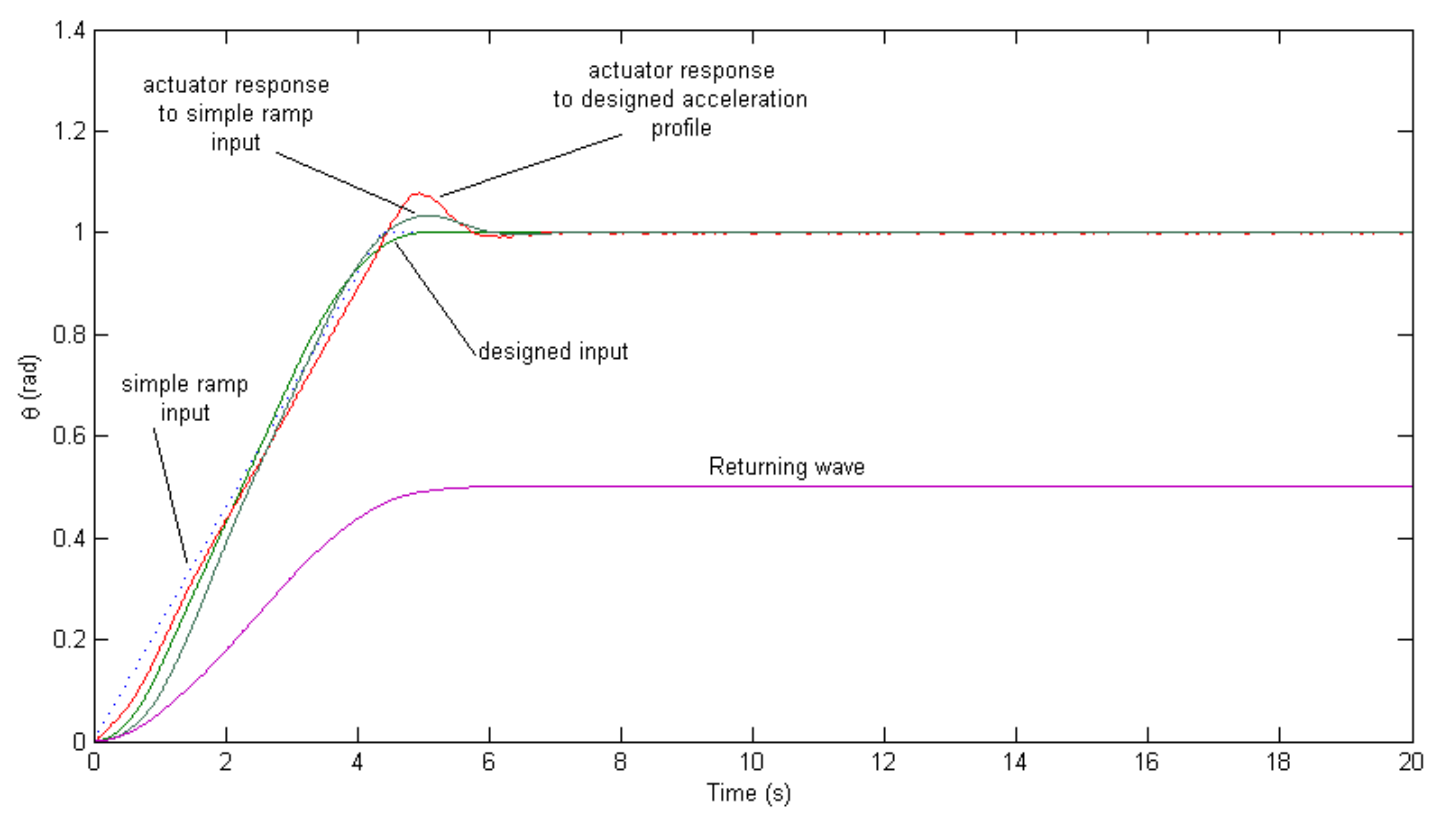


Fig.10. Rotation of mass-spring array under WBC comparing simple ramp and phased-acceleration inputs.

Using the same idea of input waveforms based on acceleration, coasting and deceleration phases, acting simultaneously on the three component motions, the residual vibrations were reduced by a factor of 10 when compared with the response to a simple ramp input with a similar transient time. The reduction was most dramatic for rotational motions alone, which also tended to have the highest residual vibration when the input was a simple ramp. These results are illustrated in Figs. 10 and 11.

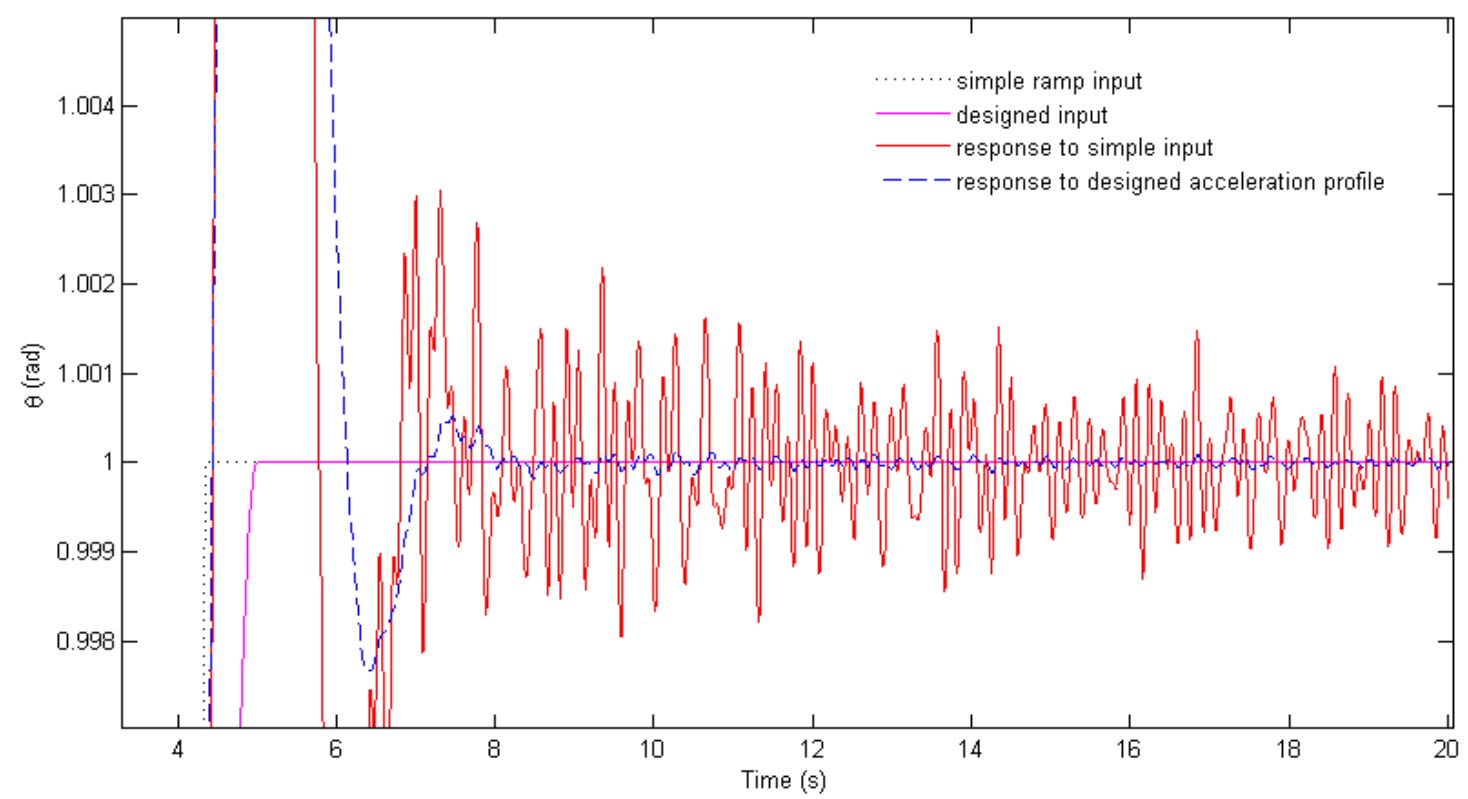

Fig.11. Illustration of much reduced residual vibration with phased-acceleration inputs.

\section{A broader perspective}

It can be claimed that, if any other technique manages to cause the actuator to achieve gross motion combined with active vibration damping, it is doing what WBC does. But while it may achieve aspects of what WBC achieves, it may require an accurate model, it may involve unnecessarily complex theories, and it may not be robust, generic or optimal. Other techniques, inevitably, use the actuator to insert momentum and energy into the flexible system, and subsequently to absorb them both, in such a way that, when all vibration has ceased, the system is at rest at the target. This is precisely what WBC does, simply, deliberately, optimally and in a generic way. (WBC is optimal in a way which can be precisely defined, within constraints which are reasonable and appropriate for real systems.) 
One possible concern that might be associated with the performance of WBC is to what extent it would cope with the systems experiencing external disturbing forces. It is well known that disturbances, whether enduring or transient, considerably complicate the flexible control problem, especially when they are unknown, unexpected or unmodelled. To address this concern, it should be noted that WBC technique has been already modified and promoted to carry out simultaneous vibration and position control of complex, under-actuated flexible systems undergoing unquantified external disturbances (see [25]). The robust control performance using the technique could be achieved by the simple measurements discussed in this paper which are taken only at the actuator. In fact, no further amendment or extra measurement from other parts of the system or details of the system properties (such as inertias, stiffnesses, dimensions, vibration modes, or damping) is needed to deal with mitigating residual vibrations in the case of presence of external disturbances.

The residual vibration problem has here been identified and studied in the context of WBC. But it seems clear that the issue is more general. Any comparable control system could leave residual vibrations on arrival at target, and almost inevitably will, if damping is light. If the only available damping method then is controlled motion of the actuator, and at the same time the actuator is required to hold the system at target, there are good reasons to propose that there is no better strategy than WBC.

The effect of WBC is to maximise the absorption (and so eliminate or minimise the reflection) of the motion arriving back to the actuator. This absorption of returning motion at the actuator is the best any controller can do, with a given actuator, in a given lumped system, once dispersion has begun. The actuator controller has to wait patiently, as it were, for the vibratory motion to arrive there, no matter how long it takes. This is because any motion of the actuator, other than to absorb returning waves, will launch a wave into the system, which in turn, as it propagates around the system, will add further scattering and dispersion, thereby further delaying the final settling.

On arrival the returning motion should meet a mechanical impedance which matches the output impedance of the flexible system, to maximise the absorption. This is what WBC does. With just one actuator on the job, nature cannot and will not be forced to go any faster, once the residual vibrations have become established.

Against the above, it might be argued that, as previously mentioned, some open-loop strategies (e.g. time-optimal bang-bang, or input shaping) can end up with precisely zero residual 
vibration, at least in theory, when perfectly implemented. But in the first place, perfect implementation is almost impossible in real systems, with real actuators, relying on modelling simplifications in the control design. Furthermore, as the order of the system grows, designing a theoretically correct input becomes more and more challenging, even assuming a perfect system model. From the wave perspective, this rapidly increasing complexity can be associated with the need to design an input which causes a succession of disturbances acting on the (dispersive) flexible system, disturbances which will superpose over time, but which must later cancel each other out perfectly and instantly, when the system is precisely at the target position. In theory and in practice, with increasing system order and complexity, this poses enormous challenges.

As an aside, if there really is a known, perfect, open-loop input which can achieve zero residual for a given real system, then WBC can also use it to design its launch wave, with the advantage of robustness to subsequent departures from this perfection, due to the self correcting effects active in WBC. This possibility is considered in [10].

\section{Concluding remarks}

The paper has studied a hitherto unexplored aspect of WBC, namely very small, slowly decaying residual vibrations which linger in the system after an otherwise completed manoeuvre. They become more obvious in higher order systems with light damping and non-uniform dynamics.

Because the residual vibrations are small, and because they are about the correct final position, they are unlikely to pose significant problems in many engineering applications. Furthermore, any internal damping (assumed negligible in this work) will accelerate their decay, especially because of their high frequency. Nevertheless, they are likely to pose a challenge in situations involving large (high order), lightly damped, non-uniform, lumped, flexible systems, such as large space structures and spacecraft observatories.

A way has been found to understand their nature and to mitigate their effects. It has been conjectured that the residual vibrations correspond to a distribution of very small amplitude, high frequency waves, remnants of repeated scattering of the launched wave throughout the system, slowly finding their way back to the actuator for absorption. Once established, the scattered waves quickly become so mixed that any hope of identifying, separating or otherwise directly controlling them seems lost.

While it therefore seems almost impossible to eliminate them without damping, a way was found nevertheless to reduce them. The method could be considered as the successive scattering 
of acceleration waves of opposite signs. In this paper the wave variable was taken to be displacement from the initial position, and the wave models were interpreted in this way. If the wave variable is instead considered to be acceleration (or velocity, or indeed force), almost identical wave models and control strategies result. Thus if, for example, instead of position control, it was desired to achieve acceleration control of an under-actuated flexible system, for example in space, one could set a reference acceleration and measure the return waves as accelerations, so doing WBC of acceleration. The same would apply to WBC of velocity or of force.

The relevance of this view here is as follows. Any motion input over time implicitly specifies position, velocity and acceleration inputs. Even though in this work the input waveforms were set as position over time, they also implicitly set acceleration waves. It then becomes possible to think that the success of the acceleration-coast-deceleration input arises from a scattering of positive acceleration waves followed by a scattering of negative acceleration waves, in approximately equal amounts, leading to partial cancelling of the lingering, distributed residuals, considered as accelerations. If the system is given a little time to settle during the coasting phase, so that gross velocities become fairly uniform, the launch of the deceleration wave finds the system not too far from its acceleration-state when the original acceleration wave was launched. So the subsequent scattering will be similar. This view also explains why the timing of the deceleration phase is not critical, provided there is a minimum delay before it begins.

In conclusion, for situations in which the small residual vibrations under WBC are a problem, in a rest-to-rest manoeuvre, their level, when the system arrives at target, can be reduced, but not eliminated, at the cost of a small lengthening of manoeuvre times. Subsequently they will then decay to zero as before.

\section{Conflict of Interest}

The authors declare that they have no conflict of interest.

\section{References}

[1] W.J. O'Connor, Wave-Based Analysis and Control of Lump-Modeled Flexible Robots. Robotics, IEEE Transactions on, 23(2), pp. 342-352 (2007).

[2] W.J. O'Connor, F. Ramos de la Flor, D.J. McKeown, V. Feliu, Wave-based control of non-linear flexible mechanical systems. Nonlinear Dynamics, 57(1), pp. 113-123 (2009). 
[3] H. Habibi, W. O'Connor, Payload motion control of rotary gantry and luffing cranes using mechanical wave concepts, Transactions of the Institute of Measurement and Control, SAGE, 2017, Vol. 39(11), pp. 1649-1662.

[4] H. Habibi, W.J. O'Connor, Wave-based control of planar motion of beam-like mass-spring arrays, Wave Motion, Volume 72 (2017), Pages 317-330.

[5] S.K. Dwivedya, P. Eberhardb, Dynamic analysis of flexible manipulators, a literature review, Mechanism and Machine Theory, 41, 7, pp 749-777, July 2006.

[6] F-Y. Wang, Y. Gao, Advanced Studies of Flexible Robotic Manipulators: Modeling, Design, Control and Applications, World Scientific Publishing Company, 2003, ISBN-13: 9789812383907

[7] R.D. Robinett III, C.R. Dohrmann, G.R. Eisler, J.T. Feddema, G.G. Parker, D.G. Wilson, D. Stokes, Flexible robot dynamics and controls, 2002, Kluwer Academic/Plenum, New York.

[8] H.G. Sage, M.F. de Mathelin, E. Ostertag, Robust control of robot manipulators: a survey, International Journal of Control, 1999, 72, 16, pp.1498-1522.

[9] M. Benosman, G.L. Vey, Control of flexible manipulators: a survey, Robotica, 22, 5, pp. 533545, Sept. 2004.

[10] W.J. O'Connor, D.J. McKeown, Time-optimal control of flexible robots made robust through wave-based feedback. ASME J Dynamic Systems, Measurement \& Control, Vol 133, Issue 1 (Jan 2011).

[11] J. Zhou, K. Zhang, and G. Hu, Wave-Based Control of a Crane System with Com-plex Loads, Journal of Dynamic Systems, Measurement, and Control, Vol. 139 (8), 081016, June 2017.

[12] S. A. Khalilpour, R. Khorrambakht, H. D. Taghirad, and P. Cardou, "Wave Based Control of A Deployable Cable Driven Robot", Robotics and Mechatronics (IcRoM) 2018 6th RSI International Conference on, pp. 166-171, 2018.

[13] J.V. de Wijdeven, O. Bosgra, Residual vibration suppression using Hankel iterative learning control, International Journal of Robust and Nonlinear Control, 2008; 18:1034-1051.

[14] G. Ma, M. Xu, B. Gao, S. Zhang and Z. Hu, The low frequency vibration control of the hoop truss structure with extended arm, Journal of Low Frequency Noise, Vibration and Active Control (2017), 36(3), 294-305.

[15] C. Conker, H. Yavuz, H. H. Bilgic, A review of command shaping techniques for elimination of residual vibrations in flexible-joint manipulators, Journal of Vibroengineering, Vol. 18, Issue 5, 2016, p. 2947-2958.

[16] L. Sirota and Y. Halevi, Free response and absolute vibration suppression of second order flexible structures - the traveling wave approach. ASME J. Vib. Acoust. (2010), 132, p. 031008.

[17] S.A. Zahedi, and V. Babitsky, Modeling of autoresonant control of a parametrically excited screen machine, Journal of Sound and Vibration, Volume 380, October 2016, pp. 78-89.

[18] Q. Zhang, Q. Lu, X. Zhang, and J. Wu, Study on Residual Vibration Suppress of a 3-DOF Flexible Parallel Robot Mechanism, Sensors (Basel). 2018 Dec; 18(12): 4145.

[19] A. Abe, Trajectory planning for residual vibration suppression of a two-link rigid-flexible manipulator considering large deformation, Mechanism and Machine Theory, Volume 44, Issue 9, September 2009, p. 1627-1639.

[20] P. Xin, J. Rong, Y. Yang, D. Xiang, Y. Xiang, Trajectory planning with residual vibration suppression for space manipulator based on particle swarm optimization algorithm, Advances in Mechanical Engineering, Volume: 9 issue: 4, April 2017. 
[21] M. Saigo and N. Tanaka, Torsional vibration suppression by wave absorption controller, Journal of Sound and Vibration (2006), 295(1-2), 317-330.

[22] K.J. Park, Flexible robot manipulator path design to reduce the endpoint residual vibration under torque constraints, Journal of Sound and Vibration 275 (2004) 1051-1068.

[23] W.J. O'Connor, Wave-like modelling of cascaded, lumped, flexible systems with an arbitrarily moving boundary, Journal of Sound and Vibration 330 (2011) pp 3070-3083.

[24] W.J. O'Connor, A. Fumagalli, Refined Wave-Based Control Applied to Nonlinear Bending and Slewing Flexible Systems. ASME Journal of Applied Mechanics, 76(4), (2009). doi:10.1115/1.3086434.

[25] W.J. O'Connor and H. Habibi, Wave-based control of under-actuated flexible structures with strong external disturbing forces, International Journal of Control, Taylor \& Francis, Vol. 88, No. 9, 1818-1829, 2015. 